\title{
Late-onset X-linked Chronic Granulomatous Disease in a Female Carrier: Therapeutic Role of Interferon-Y and Hematopoietic Stem Cell Transplantation
}

Matteo Trevisan ( $\sim$ matteo.trevisan91@gmail.com )

University of Trieste https://orcid.org/0000-0003-1897-7701

Elizabeth Kang

National Institute of Allergy and Infectious Diseases, National Institutes of Health

Francesco Salton

University Hospital of Trieste

Samuele Naviglio

Institute for Maternal and Child Health IRCCS "Burlo Garofolo"

\section{Barbara Ruaro}

University Hospital of Trieste

\section{Paola Confalonieri}

University Hospital of Trieste

\section{Erica Valencic}

Institute for Maternal and Child Health IRCCS "Burlo Garofolo"

\section{Kelly Corin}

National Institute of Allergy and Infectious Diseases, National Institutes of Health

\section{Mark Parta}

Frederick National Laboratory for Cancer Research

\section{Luigi Daniele Notarangelo}

National Institute of Allergy and Infectious Diseases, National Institutes of Health

Alberto Tommasini

University of Trieste

\section{Harry Lewis Malech}

National Institute of Allergy and Infectious Diseases, National Institutes of Health

\section{Marco Confalonieri}

University Hospital of Trieste

\section{Research Article}

Keywords: chronic granulomatous disease, primary immunodeficiency, interferon-y, hematopoietic stem cell transplantation, $\mathrm{X}$ chromosome inactivation 
Posted Date: March 23rd, 2021

DOI: https://doi.org/10.21203/rs.3.rs-344043/v1

License: (c) (i) This work is licensed under a Creative Commons Attribution 4.0 International License. Read Full License 


\section{Abstract}

X-linked Chronic Granulomatous Disease (CGD) is a rare inherited immunodeficiency characterized by early life-threatening infections from bacteria and fungi in male children. Female carriers of X-linked CGD usually do not develop any manifestations of the disease, yet in rare cases they may present with CGDrelated manifestations due to skewed $X$ chromosome inactivation, even in advanced age. Here, we report the case of a 49-year-old woman with no history of previous frequent or severe infections, who presented acutely with life-threatening bilateral pneumonia caused by Nocardia asteroides and was eventually diagnosed with late-onset $X$-linked CGD due to skewed X chromosome inactivation in white blood cells. Treatment with interferon- $\gamma$ as a rescue therapy resulted in normalization of the intensity of the oxidative burst in the residual positive cells and resolution of the infection, which was otherwise resistant to conventional treatments. After discharge, however, recurrent severe pulmonary infections despite prophylactic treatments as well as appearance of granulomatous colitis led to considering definitive treatment. Hematopoietic stem cell transplantation from unaffected HLA-identical brother using a nonmyeloablative conditioning protocol with intravenous busulfan followed by high-dose peripheral blood stem cell graft and post-transplant cyclophosphamide was successfully performed. After three years of follow-up, white blood cell chimerism remained stable with about $60 \%$ donor cells in the myeloid lineage, with no further infections and no recurrence of inflammatory bowel disease.

\section{Introduction}

Chronic granulomatous disease (CGD) is a rare inherited immunodeficiency ( 1 in 250.000 live births) [14] characterized by a defective NADPH oxidase complex, leading to an impaired respiratory burst and ineffective killing of pathogens by phagocytes (neutrophils, monocytes, and macrophages). CGD patients are especially vulnerable to infections caused by fungi or catalase-positive bacteria such as Staphylococcus aureus, Pseudomonas aeruginosa, Nocardia asteroides, and Burkholderia cepacia [1-4]. Moreover, patients can also present with clinical manifestations related to hyperinflammatory responses particularly in the lungs and gastrointestinal tract, where it can closely resemble Crohn's disease, as well as granuloma formation in various organs [5-9].

The NADPH oxidase complex subunits are encoded by five genes (CYBB, CYBA, NFC1, NFC2, and NFC4) and pathogenic mutations in any of these can cause CGD, albeit with variable phenotypes $[1,2,10]$. The most common form of CGD is caused by hemizygous mutations in the CYBB gene, encoding the gp91 ${ }^{\text {phox }}$ subunit, whereas the remaining cases are due to autosomal recessive mutations in one of the other four genes. X-linked CGD usually manifests in male children at an early age, while female carriers of a $C Y B B$ mutation generally do not show any signs of the disease. However, there are reports of females with a CGD phenotype due to progressive skewing of X-chromosome inactivation in leukocytes [11-18]. Diagnosis and treatment of these patients may be difficult. Here, we report the clinical and therapeutic course of a woman with late-onset clinical problems of CGD due to extremely skewed X chromosome lyonization, who was ultimately cured by hematopoietic stem cell transplantation (HSCT) by a matched sibling peripheral blood stem cell (PBSC) transplant after non-myeloablative conditioning. 


\section{Methods}

Dihydrorhodamine assay. Stimulation of peripheral blood sample with phorbol 12-myristate 13-acetate (PMA) induces the production of reactive oxygen species (ROS) by the NADPH oxidase enzyme activity in granulocytes. The release of ROS was revealed using dihydrorhodamine 123 (DHR123), conversion to fluorescent rhodamine 123. The fluorescence signal was read by means of a cytometer, MACSQuant Analyzer 10, and analyzed with FlowLogic software.

X-chromosome inactivation (XCl). Human androgen receptor (HUMARA) assay was used to determine the percentage of $\mathrm{X}$-chromosome inactivation $(\mathrm{XCl})$. A hypervariable CAG short tandem repeat and several methylation-sensitive restriction sites (such as Hpall) permits the discrimination between the paternally and maternally derived $\mathrm{X}$ chromosome and the selectively digestion of the active unmethylated allele. Using a subsequent polymerase chain reaction (PCR), it is possible to distinguish the active (not amplified) from the inactive alleles. The area below each peak were used to calculate the percentage of $X$ inactivation. Usually, a random $\mathrm{XCl}$ occurs in each female during embryogenesis and is estimated about $50 \%$. In case of significant deviation from this ratio, non-random or skewed X-chromosome inactivation condition occurred. The percentage of inactivation is considered unbalanced for values greater than $80 \%$ $[19,20]$.

\section{Results}

Here, we describe the case of a 49-year-old woman admitted to the Pneumology department because of severe respiratory failure due to bilateral pneumonia. Her medical history was characterized by discoid lupus and Raynaud's phenomenon, which had appeared three years before. Notably, she had no history of frequent, severe, or unusual infections. After unsuccessful treatment with broad-spectrum antibiotics, analysis of bronchoalveolar lavage fluid demonstrated Nocardia asteroides infection. Despite targeted intravenous antibiotics, she developed acute respiratory distress syndrome and bilateral pneumothorax, requiring intensive care unit admission for invasive ventilation and ultimately extracorporeal membrane oxygenation. Blood tests, lymphocytes subpopulations, and interferon-y (IFNy) levels were normal, nevertheless, DHR123 assay showed superoxide anion production only in a small portion of her neutrophils; indeed, only $6.68 \%$ of the patient's neutrophils showed were oxidase activity (healthy controls: $96-100 \%$ of neutrophils display oxidase activity by DHR assay). Moreover, the fluorescence intensity in positive cells was lower than expected (Fig. 1), suggesting a quantitative defect in superoxide anion production. Genetic analysis revealed a heterozygous mutation on CYBB gene (c.[1661_1662insT]: [=],p. [Glu555*]). X-chromosome inactivation assay performed in her peripheral white blood cells showed a skewed pattern, with a ratio of $97.8: 2.2 \%$, supporting the conclusion that almost all of her blood cells had inactivated the $\mathrm{X}$-chromosome without the mutated gene, thus confirming the functional diagnosis of $\mathrm{X}$ linked recessive CGD despite her heterozygous carrier X-CGD genotype. After one week of extracorporeal respiratory support, her condition did not improve, therefore subcutaneous IFNy was started $(200 \mu \mathrm{g} / 3$ times a week). Notably, this treatment did not influence the overall percentage of functional neutrophils at DHR123 assay, yet it led to a normalization of the fluorescence intensity in the remaining positive cells 
(Fig. 2), and was soon followed by a significant improvement of pneumonia, allowing progressive reduction of respiratory support and ultimately weaning. After hospital discharge, she developed persistent diarrhea and rectal bleeding; colonoscopy showed diffuse colitis, later histologically confirmed to be granulomatous colitis, requiring treatment with oral corticosteroids. Despite anti-microbial prophylaxis and maintenance of IFNy administration, in the following months she required multiple hospitalizations due to recurrent pulmonary infectious complications. In particular, she developed several lung infections due to Klebsiella pneumoniae, Nocardia, asteroides and Pseudomonas aeruginosa colonization, often with associated severe sepsis, ultimately requiring a resection of two pulmonary lobes of the right lung due to lung abscess with cavitation. Hematopoietic stem cell transplantation (HSCT) was recommended due to her overall worsening condition and recurrent infections. At the age of 51 years, the patient was transferred to the National Institutes of Health $(\mathrm{NIH})$ in Bethesda, Maryland, USA, where she received a healthy matched sibling HSCT graft of mobilized PBSC after non-myeloablative conditioning with intravenous busulfan and alemtuzumab. For graft versus host disease (GVHD) prophylaxis she received post-transplant cyclophosphamide on Days 3 and 4 and then sirolimus for 6 months. Just prior to initiating transplant conditioning a DHR123 assay showed showed oxidase activity in $3.1 \%$ of circulating neutrophils. At 15 days after transplantation, $99 \%$ of the early-appearing circulating neutrophils were functional neutrophils on a DHR123 assay, and neutrophil engraftment to absolute neutrophil count $>500 / \mu \mathrm{L}$ occurred on the 23rd day from HSCT. Soon after transplantation, the patient developed grade I acute skin GVHD, which was treated by topical steroids, and a self-limited asymptomatic reactivation of Cytomegalovirus and Epstein-Barr virus. No other infections or complications occurred in the follow-up. Currently, almost three- and one-half years after HSCT, her peripheral blood donor chimerism is stable ( $70 \%$ of lymphocytes and $60 \%$ of granulocytes donor derived), with $62 \%$ of the neutrophils displaying normal superoxide production capacity at DHR assay, and the patient maintains an overall good health, with resolution also of granulomatous colitis.

\section{Discussion}

Our case report highlights several peculiar aspects of late-onset X-linked CGD in female patients, underscoring the need for high clinical suspicion, but also the possibility of curative HSCT even in patients over 50 years of age. In our case, an immunodeficiency was suspected due to the occurrence of a severe and unusual infection in a woman with normal white blood cell counts and normal serum immunoglobulins without a significant previous infection history. She did have a history of discoid lupus, a finding frequently reported in female carriers of X-linked CGD [21], yet this association may go unnoticed. After secondary causes of immunodeficiency were ruled out, CGD was suspected and eventually confirmed by the finding of abnormal results at DHR testing. The presence of two functional populations of neutrophils, together with the detection of a recessive mutation in the $C Y B B$ gene confirmed the diagnosis of $\mathrm{X}$-linked recessive CGD in a woman with skewed $\mathrm{X}$ chromosome lyonization. It is already known that female carriers can present with clinical manifestations later in life due to the aging of their hematopoietic cell lineage and to progressive skewing of $X$ chromosome inactivation [11-18]. The factors leading to skewing of $X$ chromosome inactivation in some female carriers are not completely 
known. X chromosome inactivation is usually a random event, yet the presence of micro-deletions or other kinds of deleterious damages on one of the two $X$ chromosomes may lead to its preferential inactivation, leading to a skewed X inactivation [22-25]. Moreover, differential DNA methylation has been described in hematopoietic cell lineage with aging, possibly resulting in greater functional skewing [2629]. These processes may ultimately result in the selection of mutated alleles in other genes, as in the case of X-linked CGD female carriers, and thus to clinical disease when the number of functional neutrophils falls under a critical level [11-18].

Current treatment of CGD is based on prevention of severe infections, prompt and intensive antibiotic therapy, and proper treatment of granulomas and inflammatory complications. In our patient, the first episode of life-threatening pneumonia resolved only after rescue treatment with IFNY was started. IFNY enhances phagocyte activity in healthy subjects through NADPH oxidase and inducible isoform of Nitric Oxide Synthase (iNOS) transcription [30]. Even in CGD patients, IFNY increases the oxidative burst in defective neutrophils both in vitro and in vivo, although though the mechanism is not fully understood [31-34]. Although IFNy role and effectiveness for infection prophylaxis and treatment in CGD patients is still debated [35-37], our experience may suggest that it could have a greater role in heterozygous females as compared to male patients, as it could potentiate oxidase activity of the residual part of neutrophils expressing the wild type allele [38]. In fact, DHR testing in our patient showed a decreased oxidative burst also in the residual oxidase-positive neutrophils, possibly due to the concomitant sepsis, which can induce immunoparalysis of both innate and adaptive immunity [39-41]. Indeed, immunoparalysis may be a critical issue in subjects starting with already compromised immune functions.

HSCT remains the only definitive treatment for X-linked CGD to date, with a higher success rate in younger patients. Older patients, on the other hand, are more likely to develop transplant complications and have higher transplant-related mortality due to active infections, inflammation, and organ damage at the time of transplantation [42-44]. Gene therapy is also under development, with promising outcomes recently, but availability is still limited [45]. Our patient underwent HSCT because of her worsening condition despite standard prophylactic treatments (antibiotics, anti-fungal, steroids, and IFNY). She was considered at high risk for transplant-related morbidity and mortality due to her age and the presence of active infectious and inflammatory complications; therefore, a non-myeloablative conditioning regimen followed by high dose PBSC graft was used. Reduced-intensity or reduced-toxicity regimens with lowdose busulfan (AUC $45-65 \mathrm{mg} / \mathrm{L}^{*} \mathrm{~h}$ ) or treosulfan have been shown to be associated with a higher oversurvival $(>90 \%)$ and event-free-survival $(>85 \%)$ compared to myeloablative regimens, $[46,47]$ but can lead to mixed chimerism as a long-term outcome as occurred in this patient. Her donor neutrophil chimerism remains stable at $60 \%$ which appears to be sufficient to maintain her current state of good health. The $\mathrm{NIH}$ regimen incorporates low dose busulfan (30-40mg/L*h) and alemtuzumab $(1 \mathrm{mg} / \mathrm{kg})$ for patients with related donors and even lower dose busulfan (median AUC of $20-30 \mathrm{mg} / \mathrm{L}$ h) combined with low dose total body irradiation (TBI) and alemtuzumab followed by sirolimus as single agent GvHD prophylaxis [48]. For this patient, enrolled on a new protocol, she also received post-transplant cyclophosphamide along with a high dose of unmanipulated PBSC (10 million CD34 per kg) to ensure 
better engraftment but prevent GVHD. This HSCT protocol then resulted in stable donor chimerism with minimal transplant morbidity despite the patient's risk factors and age. With follow up of more than three years, this suggests that transplantation using this regimen is viable even in older patients with high risk factors.

This case demonstrates a number of points. First of all, it underscores an emerging cornerstone of clinical immunology, i.e. that even a single severe or unusual infection should raise the suspicion of a primary immune deficiency disorder, even in previously healthy adults. Secondly, IFNy may have a role as a rescue target therapy in severe infections, especially in females with X-linked CGD, as these patients still have a fraction of normal neutrophils. Lastly, HSCT with non-myeloablative conditioning with low dose busulfan and alemtuzumab, combined with an increased stem cell dose and post-transplant cyclophosphamide may represent a curative option even in high-risk patients at an advanced age.

\section{Abbreviations}

AUC: Area Under the Curve

CGD: Chronic Granulomatous Disease

DHR: Dihydrorhodamine assay

GVHD: Graft Versus Host Disease

HSCT: Hematopoietic Stem Cell Transplantation

HUMARA: Human androgen receptor

IFNY: Interferon-gamma

iNOS: inducible Nitric Oxide Synthase

NADPH: Nicotinamide Adenine Dinucleotide Phosphate

$\mathrm{NIH}$ : National Institutes of Health

PBSC: Peripheral Blood Stem Cells

PCR: Polymerase Chain Reaction

PMA: Phorbol 12-Myristate 13-Acetate

ROS: reactive oxygen species

RTE: Recent Thymic Emigrants

TBI: Total Body Irradiation 


\section{Declarations}

\section{Funding:}

This project has been funded in whole or in part with federal funds from the National Cancer Institute, National Institutes of Health, under Contract No. HHSN261200800001E and Contract No.

75N910D00024, Task Order No. 75N91019F00131. The content of this publication does not necessarily reflect the views or policies of the Department of Health and Human Services, nor does mention of trade names, commercial products, or organizations imply endorsement by the U.S. Government.

\section{Conflicts of interest/Competing interests:}

The authors declare that they have no conflict of interest.

\section{Availability of data and material:}

Not applicable

\section{Code availability:}

Not applicable

\section{Authors' contributions:}

All authors contributed to the study conception and design. The first draft of the manuscript was written by MT and EMK. FS, BR, PC, CK, MP, EMK, AT, HLM, and MC cared for the patient. Material preparation, clinical data collection and analysis were performed by EV, SN, MP and AT. LDN, AT, HLM and MC reviewed and improved the final draft. All authors read and approved the final manuscript.

\section{Consent to publish:}

The patient's consent to publish was obtained.

\section{References}

1. Segal BH, Leto TL, Gallin JI, Malech HL, Holland SM. Genetic, Biochemical, and Clinical Features of Chronic Granulomatous Disease. Medicine (Baltimore). 2000;79(3):170-200.

2. Rider NL, Jameson MB, Creech CB. Chronic Granulomatous Disease: Epidemiology, Pathophysiology, and Genetic Basis of Disease. J Pediatric Infect Dis Soc. 2018;7:S2-S5.

3. Seger RA. Chronic Granulomatous Disease. In: Encyclopedia of Immunobiology. Vol 5. Elsevier; 2016:485-493. 
4. Marciano BE, Spalding C, Fitzgerald A, et al. Common severe infections in chronic granulomatous disease. Clin Infect Dis. 2015;60(8):1176-1183.

5. Marks DJB, Miyagi K, Rahman FZ, Novelli M, Bloom SL, Segal AW. Inflammatory bowel disease in CGD reproduces the clinicopathological features of Crohn's disease. Am J Gastroenterol. 2009;104(1):117-124.

6. Arnold DE, Heimall JR. A Review of Chronic Granulomatous Disease. Adv Ther. 2017;34(12):25432557.

7. Rosenzweig SD. Inflammatory manifestations in chronic granulomatous disease (CGD). J Clin Immunol. 2008;28 Suppl 1:S67-72.

8. Marciano BE, Rosenzweig SD, Kleiner DE, et al. Gastrointestinal involvement in chronic granulomatous disease. Pediatrics. 2004;114(2):462-468.

9. De Ravin SS, Naumann N, Cowen EW, et al. Chronic granulomatous disease as a risk factor for autoimmune disease. J Allergy Clin Immunol. 2008;122(6):1097-1103.

10. Stasia MJ, Li XJ. Genetics and immunopathology of chronic granulomatous disease. Semin Immunopathol. 2008;30(3):209-235.

11. Nelson KS, Lewis DB. Adult-onset presentations of genetic immunodeficiencies: genes can throw slow curves. Curr Opin Infect Dis. 2010;23(4):359-364.

12. Anderson-Cohen M, Holland SM, Kuhns DB, et al. Severe phenotype of chronic granulomatous disease presenting in a female with a de novo mutation in gp91-phox and a non familial, extremely skewed X chromosome inactivation. Clin Immunol. 2003;109(3):308-317.

13. Wolach B, Scharf Y, Gavrieli R, de Boer M, Roos D. Unusual late presentation of X-linked chronic granulomatous disease in an adult female with a somatic mosaic for a novel mutation in CYBB. Blood. 2005;105(1):61-66.

14. Berrón-Ruiz L, Morín-Contreras A, Cano-García V, et al. Detection of inheritance pattern in thirty-three Mexican males with chronic granulomatous disease through 123 dihydrorhodamine assay. Allergol Immunopathol (Madr). 2014;42(6):580-585.

15. Amaral JB, Paiva AA, Ramos FV, Stasia MJ, Lemos SG. X-linked chronic granulomatous disease in a female carrier with novel pathogenic mutation and skewed X-inactivation. Ann Allergy Asthma Immunol. 2018;120(3):328-329.

16. Gono T, Yazaki M, Agematsu $\mathrm{K}$, et al. Adult onset $\mathrm{X}$-linked chronic granulomatous disease in a woman patient caused by a de novo mutation in paternal-origin CYBB gene and skewed inactivation of normal maternal X chromosome. Intern Med. 2008;47(11):1053-1056.

17. Eguchi M, Yagi C, Tauchi H, Kobayashi M, Ishii E, Eguchi-Ishimae M. Exon skipping in CYBB mRNA and skewed inactivation of $\mathrm{X}$ chromosome cause late-onset chronic granulomatous disease. Pediatr Hematol Oncol. 2018;35(5-6):341-349.

18. Marciano BE, Zerbe CS, Falcone EL, et al. X-linked carriers of chronic granulomatous disease: Illness, Iyonization, and stability. J Allergy Clin Immunol. 2018;141(1):365-371. 
19. Amos-Landgraf JM, Cottle A, Plenge RM, Friez M et al. X chromosome-inactivation patterns of 1,005 phenotypically unaffected females.Am J Hum Genet. 2006; 79:493-499.

20. Plenge RM, Stevenson RA, Lubs HA et al.Skewed X-chromosome inactivation is a common feature of X-linked mental retardation disorders. Am J Hum Genet. 2002; 71:168-173.

21. Sillevis Smitt JH, Weening RS, Krieg SR, Bos JD. Discoid lupus erythematosus-like lesions in carriers of X-linked chronic granulomatous disease. Br J Dermatol. 1990 May;122(5):643-50.

22. Shvetsova E, Sofronova A, Monajemi R, et al. Skewed X-inactivation is common in the general female population. Eur J Hum Genet. 2019;27(3):455-465.

23. Tonon L, Bergamaschi G, Dellavecchia C, et al. Unbalanced X-chromosome inactivation in haemopoietic cells from normal women. Br J Haematol. 1998;102(4):996-1003.

24. Vallot $C$, Ouimette $J-F$, Rougeulle $C$. Establishment of $X$ chromosome inactivation and epigenomic features of the inactive X depend on cellular contexts. Bioessays. 2016;38(9):869-880.

25. Lu Z, Carter AC, Chang HY. Mechanistic insights in X-chromosome inactivation. Philos Trans $R$ SoC Lond B Biol Sci. 2017;372(1733). doi:10.1098/rstb.2016.0356

26. El Kassar N, Hetet G, Brière J, Grandchamp B. X-chromosome inactivation in healthy females: incidence of excessive lyonization with age and comparison of assays involving DNA methylation and transcript polymorphisms. Clin Chem. 1998;44(1):61-67.

27. Hatakeyama C, Anderson CL, Beever CL, Peñaherrera MS, Brown CJ, Robinson WP. The dynamics of X-inactivation skewing as women age. Clin Genet. 2004;66(4):327-332.

28. Busque L, Paquette $Y$, Provost $S$, et al. Skewing of $X$-inactivation ratios in blood cells of aging women is confirmed by independent methodologies. Blood. 2009;113(15):3472-3474.

29. Kristiansen $M$, Knudsen GPS, Bathum L, et al. Twin study of genetic and aging effects on $X$ chromosome inactivation. Eur J Hum Genet. 2005;13(5):599-606.

30. Ellis TN, Beaman BL. Interferon-gamma activation of polymorphonuclear neutrophil function. Immunology. 2004;112(1):2-12.

31. Gallin JI. Interferon-gamma in the treatment of the chronic granulomatous diseases of childhood. Clin Immunol Immunopathol. 1991;61(2 Pt 2):S100-5.

32. Jackson SH, Miller GF, Segal BH, et al. IFN-gamma is effective in reducing infections in the mouse model of chronic granulomatous disease (CGD). J Interferon Cytokine Res. 2001;21(8):567-573.

33. Ezekowitz RAB, Orkin SH, Newburger PE. Recombinant interferon gamma augments phagocyte superoxide production and X-chronic granulomatous disease gene expression in X-linked variant chronic granulomatous disease. J Clin Invest. 1987;80(4):1009-1016.

34. Ishibashi F, Mizukami T, Kanegasaki S, et al. Improved superoxide-generating ability by interferon gamma due to splicing pattern change of transcripts in neutrophils from patients with a splice site mutation in CYBB gene. Blood. 2001;98(2):436-441.

35. A controlled trial of interferon gamma to prevent infection in chronic granulomatous disease. The International Chronic Granulomatous Disease Cooperative Study Group. N Engl J Med. 
1991;324(8):509-516.

36. Marciano BE, Wesley R, De Carlo ES, et al. Long-term interferon-gamma therapy for patients with chronic granulomatous disease. Clin Infect Dis. 2004;39(5):692-699.

37. Martire B, Rondelli R, Soresina A, et al. Clinical features, long-term follow-up and outcome of a large cohort of patients with Chronic Granulomatous Disease: an Italian multicenter study. Clin Immunol. 2008;126(2):155-164.

38. Åhlin A, Elinder G, Palmblad J. Dose-Dependent Enhancements by Interferon-y on Functional Responses of Neutrophils From Chronic Granulomatous Disease Patients. Blood. 1997;89(9):33963401.

39. Delano MJ, Thayer T, Gabrilovich S, et al. Sepsis induces early alterations in innate immunity that impact mortality to secondary infection. J Immunol. 2011;186(1):195-202.

40. Delano MJ, Ward PA. Sepsis-induced immune dysfunction: can immune therapies reduce mortality? J Clin Invest. 2016;126(1):23-31.

41. Payen D, Faivre V, Miatello J, et al. Multicentric experience with interferon gamma therapy in sepsis induced immunosuppression. A case series. BMC Infect Dis. 2019;19(1):931.

42. Seger RA, Gungor T, Belohradsky BH, et al. Treatment of chronic granulomatous disease with myeloablative conditioning and an unmodified hemopoietic allograft: a survey of the European experience, 1985-2000. Blood. 2002;100(13):4344-4350. doi:10.1182/blood-2002-02-0583

43. Connelly JA, Marsh R, Parikh S, Talano J-A. Allogeneic Hematopoietic Cell Transplantation for Chronic Granulomatous Disease: Controversies and State of the Art. J Pediatric Infect Dis Soc. 2018;7(suppl_1):S31-S39. doi:10.1093/jpids/piy015

44. Parta M, Hilligoss D, Kelly C, et al. Failure to Prevent Severe Graft-Versus-Host Disease in Haploidentical Hematopoietic Cell Transplantation with Post-Transplant Cyclophosphamide in Chronic Granulomatous Disease. J Clin Immunol. 2020;40(4):619-624.

45. Kohn DB Booth C, Kang EM, et al. Lentiviral gene therapy for X-linked chronic granulomatous disease. Nat Med. 2020 Feb 26(2): 200-206.

46. Güngör T, Teira P, Slatter M, et al. Reduced-intensity conditioning and HLA-matched haemopoietic stem-cell transplantation in patients with chronic granulomatous disease: a prospective multicentre study. Lancet. 2014;383(9915):436-448.

47. Morillo-Gutierrez B, Beier R, Rao K, et al. Treosulfan-based conditioning for allogeneic HSCT in children with chronic granulomatous disease: a multicenter experience. Blood. 2016;128(3):440-448.

48. Parta M, Kelly C, Kwatemaa N, et al. Allogeneic Reduced Intensity Hematopoietic Stem Cell Transplantation for Chronic Granulomatous Disease: a Single-Center Prospective Trial. J Clin Immun. 2017;37:548-558.

\section{Figures}




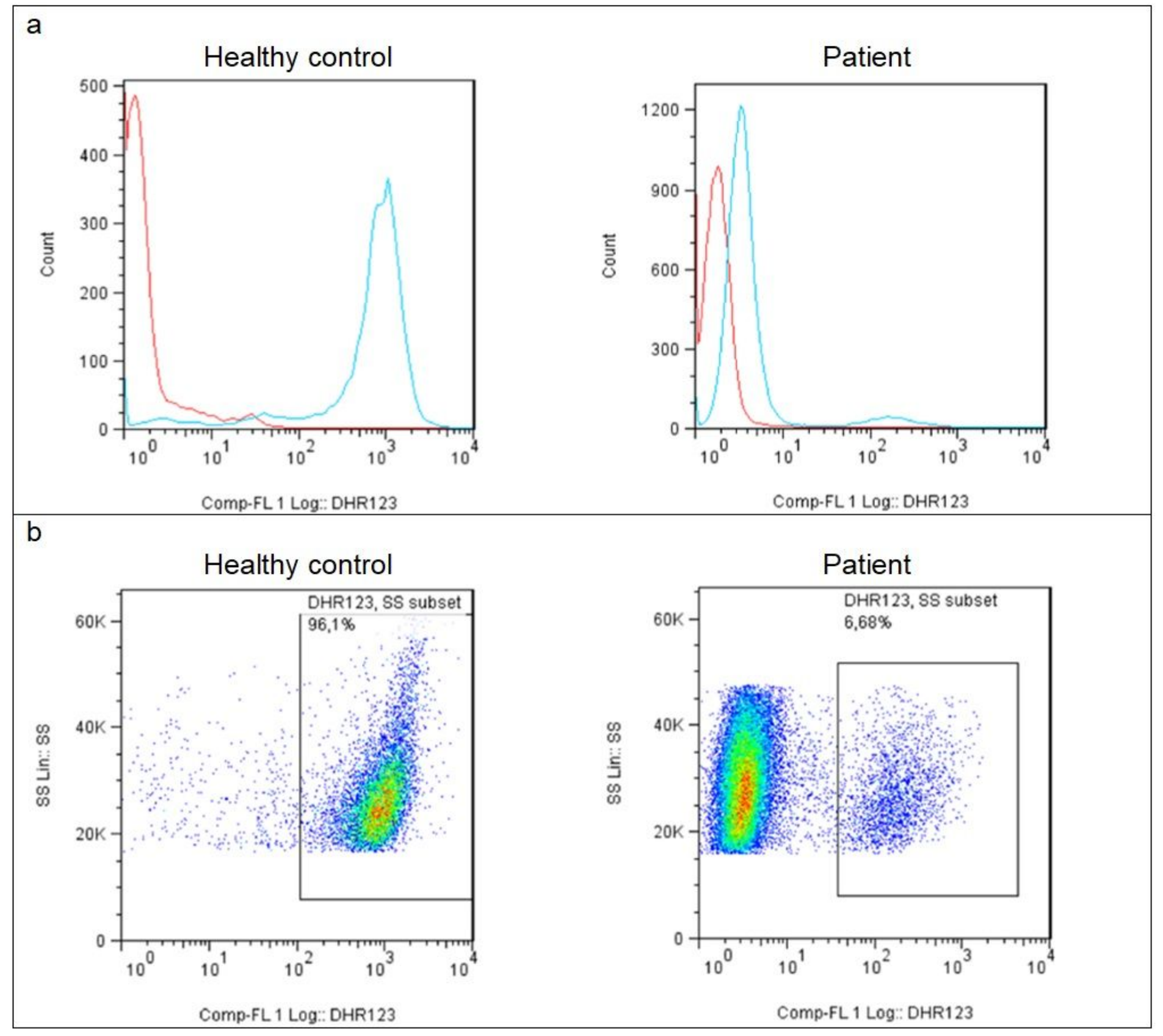

Figure 1

Dihydrorhodamine assay (DHR). Histogram graphs in Panel A show a low superoxidase production by the patient's neutrophils rather than control after PMA treatment (blue line); red line represents the untreated samples. Dot plot graphs in Panel B just to better display the low percentage of functional neutrophils (6.68\%). 


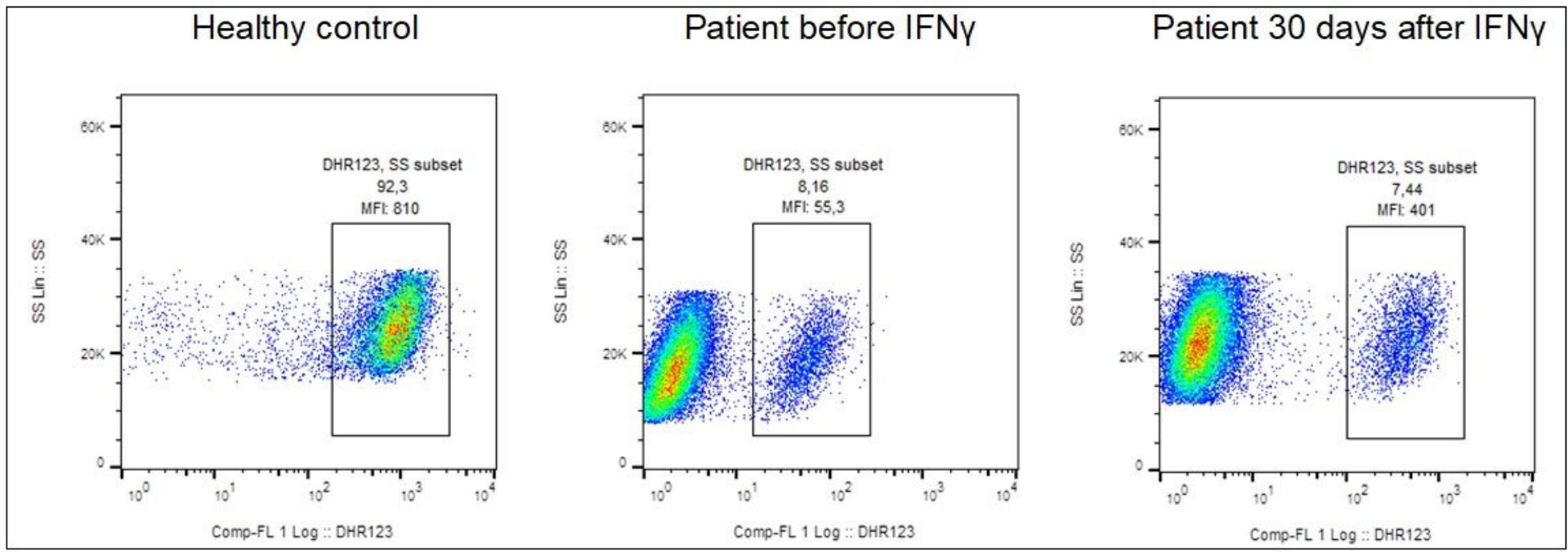

Figure 2

Dihydrorhodamine assay (DHR) before and after IFNy treatment. Comparison of the fluorescence intensity of rhodamine 123 in functional neutrophils before and after IFNy administration allows to observe an increase of median fluorescence intensity (MFI) values after the treatment (55,3 vs 401) without a corresponding rise in percentage of cells with oxidase activity. 